\title{
Experimental validation of morphology simulation in glass fibre reinforced polycarbonate discs
}

\author{
N. M. Neves, A. J. Pontes and A. S. Pouzada \\ Departamento de Engenharia de Polímeros, Universidade do Minho, \\ Campus de Azurém, 4800-058 Guimarães \\ Portugal
}

\begin{abstract}
As assessment is made between Moldflow simulations and experimentally determined fibre orientation distributions at three points along the flow path and 12 layers across the thickness. The material used is a $10 \%$ weight short glass fibre reinforced plycarbonate. With this material the physical interaction between fibres during flow is minimised. Centre gated circular discs, where both the shear and the extensional flows are present, were produced over a range of moulding conditions to analyse the effect of flow rate and melt temperature upon the fibre orientation.

The fibre orientation was measured using image analysis tools in images obtained by reflection microscopy of polished sections, using the method proposed by Bay. The measurements were made in 12 layers across the thickness.
\end{abstract}

\section{Introduction}

Thermoplastics reinforced with short glass fibres have been increasingly used to produce engineering parts for structural applications. The use of short fibres has the advantage of achieving substantial stiffening without compromising significantly the processability of the materials.

The melt flow inside cold moulds results in a complex thermomechanical process changing from point to point within the parts and through thickness. When the flow is dominated by shear stresses the fibres tend to align in the flow direction, whereas a predominantly extensional flow tends to align the fibres in the principal extension direction. The resulting morphology is complex and is related to those alignment effects and to the variation of cooling rate throughout the parts.

Tucker et al. (1994) proposed a model to predict the fibre orientation in injection mouldings based in the Jeffery's theory, where the interaction between neighbouring fibres during flow was accounted for. That model has been implemented in commercial packages like Moldflow (Moldflow Pty, Kilsyth, Australia).

\section{Modelling the fibre orientation in mouldings}

The earliest work on modelling of fibre orientation in shear flow was done by Jeffery, who established the basis of the motion of ellipsoidal particles immersed in a viscous fluid. That first approach was based in very dilute suspensions, mainly with one single fibre. Cox extended that analysis to long particles in viscous fluids. Batchelor studied the forces acting in particles immersed in dilute and semi-dilute (or semi-concentrated) suspensions followed Cox work.

Recently, Folgar, Bay ${ }^{1}$, Tucker and Akbar attempted to overcome the difficulties in predicting fibrefibre interactions applying some phenomenological parameters to the Jeffery's theory. To date there is no complete theory to predict the fibre orientation resulting from the injection moulding process. Nevertheless, Advani proposed models that allow the study of the rheological and mechanical properties of short fibre reinforced injection moulded composites.

The fibre orientation prediction is normally decoupled from the flow simulation. This means that it is assumed that the fibre orientation does not affect the flow dynamics nor the viscosity field. 
Journal Reinforced Plastics and Composites, vol. 20: $\mathrm{n}^{\circ} 6$ (2001), p.452-465.

\section{The Jeffery model}

Jeffery model predicts that a single ellipsoidal particle in a simple shear flow will undergo a periodic rotation. If the flow field is described by:

$$
v_{3}=\dot{\gamma} x_{1} ; v_{1}=v_{2}=0
$$

where $\dot{\gamma}$ is the shear rate, the fibre orientation is obtained from the angles $\theta$ and $\phi$. Those angles are calculated using the following equations:

$$
\tan \phi=\frac{C_{t} r_{e}}{\sqrt{\cos ^{2} \theta+r_{e}^{2} \sin ^{2} \theta}} ; \operatorname{cotg} \theta=r_{e} \tan \left(\frac{2 \pi t_{1}}{T_{r}}+k_{1}\right)
$$

where $T_{r}$ is the period of rotation given by

$$
T_{r}=\frac{2 \pi}{\dot{\gamma}}\left(r_{e}+\frac{1}{r_{e}}\right)
$$

$r_{e}$ being the equivalent ellipsoidal axis ratio, length/diameter. The orbit constant $C_{t}$ and the phase $k_{1}$ are determined by the initial orientation of the particle. If the motion of the particle is traced in the space, the particle will continually retrace the same path. Hence, these paths are called the Jeffery's orbits (Figure 1).
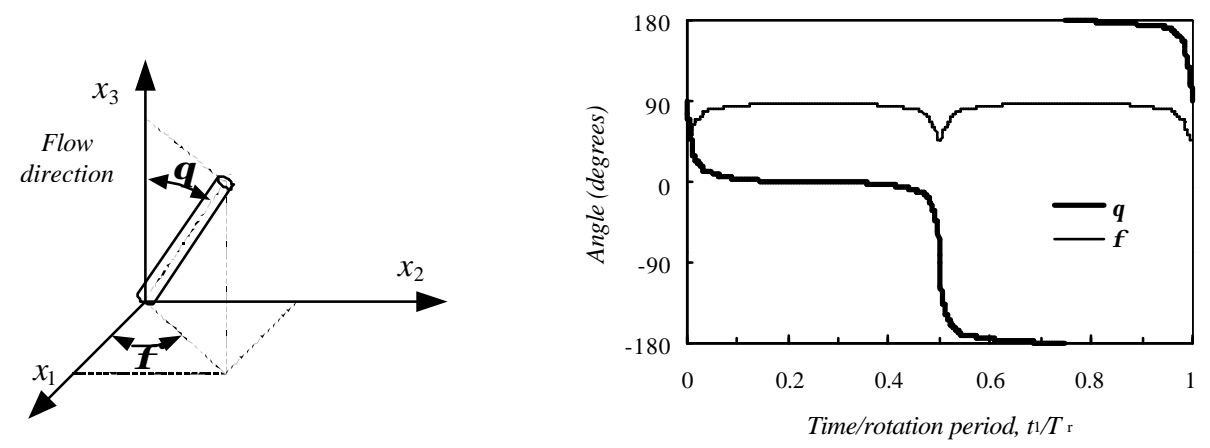

Figure 1 Periodic rotation of a single fibre immersed in a flowing liquid

It has been verified that the particles in shearing flows tend to align in the direction of the flow. In the case of stretching flows, the fibres tend to align in the direction of the greatest stretching. That direction of stretching does not need to be the direction of flow. In radial and divergent flow, sufficiently far from the injection point, the direction of greatest stretching is perpendicular to the flow and the fibres tend to align in that direction.

The predictions of fibre orientation based on the Jeffery model are qualitatively correct for common short fibre composites, but do not have useful quantitative accuracy. However, careful laboratory experiments satisfying the Jeffery assumptions, match the theory perfectly. Therefore, Tucker suggested that any deviations from the Jeffery equations must result from violation of some of his assumptions.

\section{The Folgar-Tucker model}

Some commercially available simulation packages (e.g. C-Mold or Moldflow) implement a model developed by Folgar and Tucker to predict the fibre orientation in short fibre thermoplastics. The model is valid if the following conditions are verified:

1. The fibres are rigid cylinders, uniform in length and diameter. 
Journal Reinforced Plastics and Composites, vol. 20: $\mathrm{n}^{\circ} 6$ (2001), p.452-465.

2. The fibres are sufficiently large to cause the Brownian motion to be negligible.

3. The matrix fluid is so viscous that both particle inertia and particle buoyancy are negligible.

4. The centres of mass of the particles are randomly distributed.

5. There are no external forces or torques acting onto the suspension.

The model is based in a phenomenological approach. The continuity equation ca be solved, and a statistical approach to determine the evolution of the probability distribution function for fibre orientation with time adopted. If the only variable of interest is the angle $\phi$, it results in:

$$
\dot{\phi}=\left[-\sin \phi \cos \phi \frac{\partial v_{x}}{\partial x}-\sin ^{2} \phi \frac{\partial v_{x}}{\partial y}+\cos ^{2} \phi \frac{\partial v_{y}}{\partial x}+\sin \phi \cos \phi \frac{\partial v_{x}}{\partial y}\right]-\frac{C_{I} \dot{\gamma}}{\psi_{\phi}} \frac{\partial \psi_{\phi}}{\partial \phi}
$$

The term inside the square brackets is identical to that of the Jeffery model when $r$ tends to infinity. The last term represents the effect of the interactions between fibres. Here, $\gamma$ is the magnitude of the strain rate tensor and $C_{I}$ is an empirical interaction coefficient. This coefficient is only dependent on the fibre aspect ratio, $r$, and on the fibre volume fraction, $V_{f}$. Therefore it is an intrinsic property of the suspension. However, without a detailed model for the interactions between fibres, there is no way to predict $C_{I}$, which needs to be determined experimentally. Nevertheless, Tucker suggested an empirical expression to calculate that coefficient:

$$
C_{I}=0.0184 \exp \left(-0.7148 V_{f} \frac{L}{d}\right)
$$

That equation is valid in the concentrated regime $\left(V_{f .} L d>1\right)$.

The Folgar-Tucker model leads to a number of conclusions that were confirmed experimentally:

1. The final state of orientation is dependent on the amount of strain experienced during the flow, but it is independent from the rate of deformation.

2. For any given steady flow (simple shear, elongational flow) it exists a steady state distribution of fibre orientation that is achieved regardless of the initial conditions.

3. Elongational flows produce a more highly aligned steady state than shear flows.

\section{Tensors of fibre orientation}

To obtain a tensor description of the fibre orientation of a specimen it is necessary to characterise its state of orientation. In the following, it is assumed that the fibres are rigid cylinders, all having the same length and diameter, and being equally dispersed in the whole volume (uniform concentration of fibres). If these assumptions are valid, then the orientation of a single fibre can be described by the inplane angle $\phi$, and the out-of-plane angle $\theta$, as shown in Figure 2.

The most general description of fibre orientation in a specimen is the orientation distribution function, $\psi$. At any point in the space, the state of orientation can be described by an orientation distribution function $\psi(\phi, \theta)$. This function is defined according to the condition that the probability of having a fibre in the domain $\left[\phi_{1} ; \phi_{1}+\delta \phi\right] \times\left[\theta_{1} ; \theta_{1}+\delta \theta\right]$, is given by:

$$
P(\theta 1 \leq \theta \leq \theta 1+\delta \theta, \phi 1 \leq \phi \leq \phi 1+\delta \phi)=\psi(\theta, \phi) \sin (\theta) d \theta d \phi
$$

An equivalent way to describe the orientation consists in associating a unit vector $\boldsymbol{p}$ with the fibre, as shown in Figure 2. Note that $x_{1}$ represents the flow direction axis, $x_{2}$ is the transverse flow direction axis and $x_{3}$ represents the thickness direction axis. Thus, the orientation distribution function can be written as a function $\psi(\boldsymbol{p})$ depending on the components of the unit vector $\boldsymbol{p}$ : 


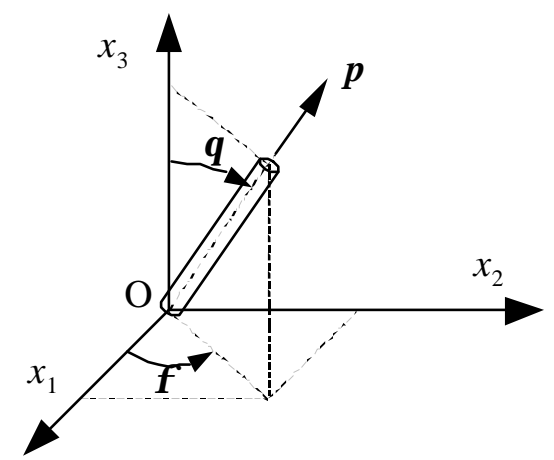

Figure 2 Characterisation of the fibre orientation in a co-ordinate system

The components of $\boldsymbol{p}$, when viewed projected onto the $x_{1}-x_{2}$ plane, are related to $\phi$ and $\theta$, according to the following equations:

$$
\begin{aligned}
& p_{1}=\sin \theta \cos \phi \\
& p_{2}=\sin \theta \sin \phi \\
& p_{3}=\cos \theta
\end{aligned}
$$

The fact of $\boldsymbol{p}$ being a unit vector implies that only two of its components are independent. The summation convention implies summation over all values of any repeated index. This convention, together with the fact that $\boldsymbol{p}$ is a unit vector, can be written as:

$$
p_{i} p_{i}=\sum_{i=1}^{3} p_{i} p_{i}=p_{1}^{2}+p_{2}^{2}+p_{3}^{2}=1
$$

In experimental terms, the tensor of fibre orientation can be obtained if a statistically significant number of fibres in a specimen is characterised in terms of the angles $\phi$ and $\theta$. From that information and keeping in mind the assumption of uniform fibre length, the second and fourth-order tensors of fibre orientation can be defined from the components of the unit vector $\boldsymbol{p}$ associated with each fibre. $\mathrm{Bay}^{2}$ suggested the equation for the calculation of the tensors:

$$
a_{i j}=\frac{\sum_{n} p_{i_{n}} p_{j_{n}} F_{n}}{\sum_{n} F_{n}}
$$

The parameter $F_{n}$ is a weighting function that corrects the bias resulting from the lower probability of a fibre lying parallel to the sectioning plane appear in the image. In this case $F_{n}$ was taken as $(\cos \theta)^{-1}$ as proposed by Bay ${ }^{2}$. Möginger et al. proposes and discusses other weighting functions.

There is some uncertainty about the measurement of $\theta$, since the same ellipse would appear if the fibre is rotated $180^{\circ}$ around the in plane angle $\phi$. One solution for this problem is the successive polishing of the same specimen at various depths, but some difficulties can arise to determine the position of the fibres at each polishing stage.

\section{Experimental}

The nominal $1.5 \mathrm{~mm}$ thick and $114 \mathrm{~mm}$ diameter disc, sprue gated in the centre, is represented in Figure 3. The actual dimensions of the moulded discs depend on the processing conditions. The sprue is conical in shape with a starting diameter of $5.5 \mathrm{~mm}$, and a final diameter of $7.5 \mathrm{~mm}$, and length of $65 \mathrm{~mm}$. Those dimensions can be considered too large when compared with the dimensions of the disc, but were chosen to minimise the initial fibre orientation. 


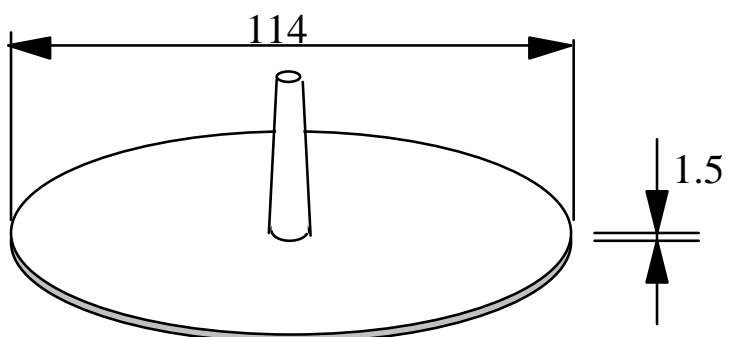

Figure 3 Nominal dimensions of the disc

A $10 \%$ in weight glass fibre reinforced polycarbonate grade (Lexan $500 R$ from General Electric Plastics) was used. This material is compounded with a release agent and a flame-retardant additive. In this case, seven moulding conditions were chosen. The mould temperature was kept at $100{ }^{\circ} \mathrm{C}$, the melt temperature and the flow rate being varied. This moulding programme enabled the study of both the effect of the flow rate at the two extremes of melt temperature, and the effect of the melt temperature at an intermediate value of flow rate (Table 1).

Table 1 Moulding programme and codes for Lexan $500 \mathrm{R}$ discs

\begin{tabular}{|c|c|c|c|}
\hline Code & $\begin{array}{c}\text { Melt } \\
\text { temperature } \\
\left({ }^{\circ} \mathrm{C}\right)\end{array}$ & $\begin{array}{c}\text { Injection flow } \\
\text { rate } \\
\left(\mathrm{cm}^{3} / \mathrm{s}\right) \\
\end{array}$ & $\begin{array}{c}\text { Mould } \\
\text { temperature } \\
\left({ }^{\circ} \mathrm{C}\right)\end{array}$ \\
\hline D280.10 & \multirow{3}{*}{280} & 9.7 & \multirow{7}{*}{100} \\
\hline D280.14 & & 13.8 & \\
\hline D280.32 & & 32.2 & \\
\hline D300.14 & 300 & 13.8 & \\
\hline D320.10 & \multirow{3}{*}{320} & 9.7 & \\
\hline D320.14 & & 13.8 & \\
\hline D320.32 & & 32.2 & \\
\hline
\end{tabular}

Two discs per moulding condition were randomly selected for fibre orientation measurements. The specimens used were cut at 20,35 and $50 \mathrm{~mm}$ from the gate, as shown in Figure 4. The cross-sections were cut on the plane perpendicular to the plane of the disc, in the radial direction. These crosshatched sections are shown in Figure 4.

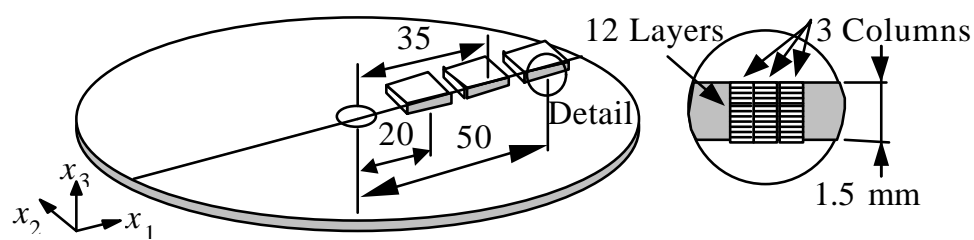

Figure 4 Specimens cut from the discs and measurement matrix

The measurements were made along three columns through the depth of the section (thickness of the disc) to assess the sampling error. Each column was divided into 12 rows or layers, each one with $1 / 12^{\text {th }}$ of the disc thickness (see detail in Figure 4). The measurements in various columns enable the evaluation of the sampling error according to the method outlined by $\mathrm{Bay}^{2}$.

The sections of the fibres appear in the image as circles if the fibres are aligned in a direction perpendicular to the sectioning surface, rectangles if the fibre axis is parallel to it, or ellipses otherwise (Figure 5). 


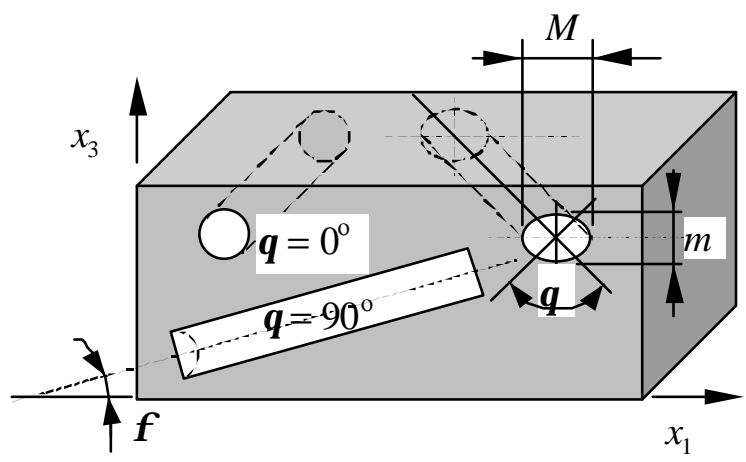

Figure 5 Fibre sections in a polishing surface, $\theta$ is the out-of-plane angle

The out-of-plane orientation angle $\theta$ is derived from the major and minor axes of the ellipse, $M$ and $m$, as

$$
\boldsymbol{\theta}=\arccos \left(\frac{m}{M}\right)
$$

The results reported here refer to three-dimensional data obtained from measurements on a single plane. This implies that ambiguity in the out-of-diagonal tensor components will appear in the results. The in-plane orientation is determined by the angle $\phi$ defined by the major axis of the ellipse and the preselected reference axes (Figure 5). These angles, $\theta$ and $\phi$, can be determined either by manually digitising the co-ordinates of the endpoints of the major and minor axes of the ellipses or by automatic image analysis. In this work, manual digitisation was used because of the poor contrast between fibre and matrix.

The relevant components of the unit vector $\boldsymbol{p}$, required for the calculation of the orientation tensor depend on the sectioning plane. Equations 6 to 8 can be applied if the specimen section is in the $x_{1}-x_{2}$ plane. However, if the section is in the $x_{1}-x_{3}$ plane as was done in this work, the out-of-sectioningplane angle, $\theta$, and the in-sectioning-plane angle, $\phi$, must be coherent to the fixed co-ordinate system. Thus, the components of the unit vector, $p_{i}$, were calculated using the following equations:

Table 2 Unit vector component definitions according to the $x_{1}-x_{3}$ sectioning plane

$x_{1}-x_{3}$ sectioning plane
$p_{1}=\sin \theta \cos \phi$
$p_{2}=\cos \theta$
$p_{3}=\sin \theta \sin \phi$

The elements of the second-order tensor that describe the fibre orientation can be calculated from the unit vector components, $p_{i}$, using equation 6.

\section{Moldflow modelling}

A 396-element model of the disc was created in Moldflow (Figure 6). The moulding parameters were introduced as in the experiments. The fibre data such as number average fibre length, diameter and averaged volume fraction were determined experimentally as $238 \mu \mathrm{m}, 14 \mu \mathrm{m}$ and $5.8 \%$, respectively.

The interaction coefficient value (necessary to account for the interaction between neighbouring fibres) used in the simulations was 0.01 as resulting from Tucker equation 4. 


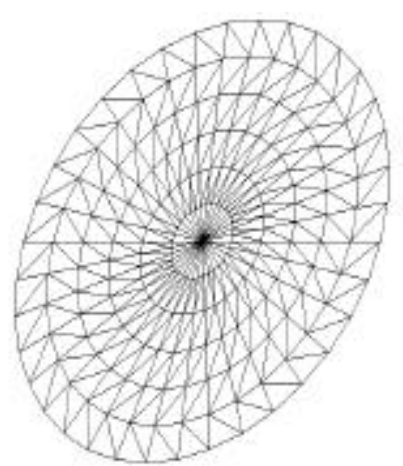

Figure 6 Moldflow model of the disc

\section{Results and discussion}

The results are presented in terms of the element $a_{11}$ of the tensor of fibre orientation. This tensor component characterises the degree of alignment of the fibres in the flow direction. The Modflow fibre software predicts a predominantly planar fibre orientation distribution with very small values for the out-of-plane component of the tensor of fibre orientation $\left(a_{33}=0.01\right)$. Thus, the transverse-flowdirection fibre orientation at any point in the results may be seen as the difference between $a_{11}$ and the unit.

The variation of fibre orientation along the flow path can be observed in Figure 7-a, -b and -c. The patterns of fibre orientation through-thickness obtained from Moldflow modelling are in accordance with expectations. A core region can be defined, where the fibre orientation is predominant in the tangential direction (low $a_{11}$ ). At the skin, a thin layer appears and is characterised by in plane random fibre orientation $\left(a_{11} \approx a_{22} \approx 0.5\right)$. At some distance from the wall, a maximum of fibre orientation in flow direction can be observed. The experimental results show less defined layering throughthickness.

A trend to obtain higher fibre orientation in the flow direction can be observed in the Moldflow simulation results, being more visible close to the through-thickness maximum value. That trend is less evident in the experimental results, where the through-thickness fibre orientation in flow direction does not show maxima as high as those obtained in the simulation.

The error bars shown in Figure 7-a, -b and -c are typical of the results obtained. It can be observed that the errors are higher close to the wall than in the midplane of the mouldings. This may be a result of the smaller number of fibres appearing at those locations. Those fibres have lower probability of appearing in the sectioning plane.

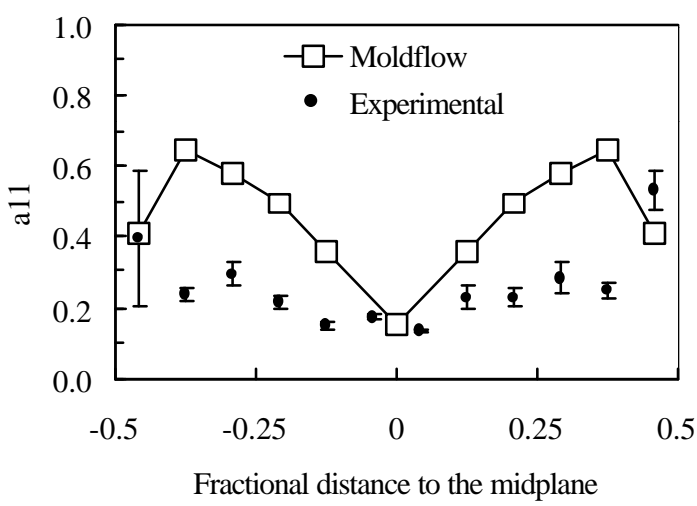

a)

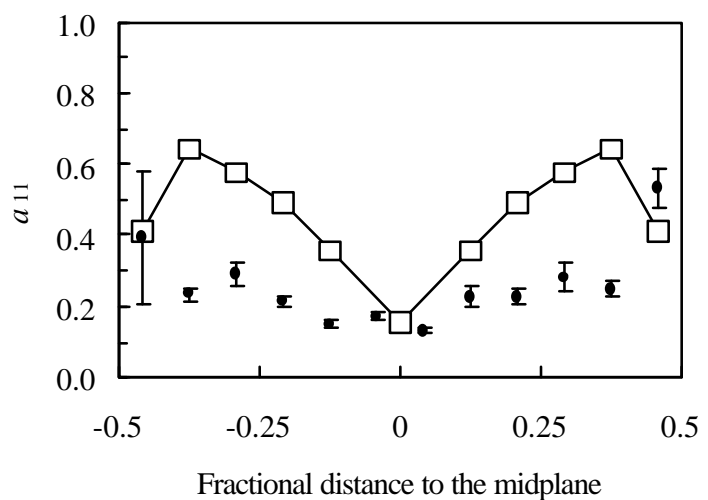

b) 


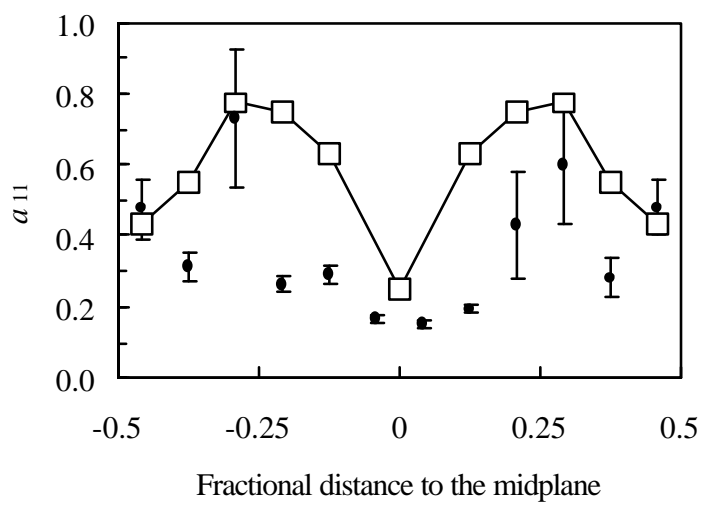

c)

Figure 7 Fibre orientation in flow direction at $280^{\circ} \mathrm{C}$ melt temperature and $10 \mathrm{~cm}^{3} / \mathrm{s}$ flow rate, at three distances from the gate. a) $20 \mathrm{~mm}$, b) $35 \mathrm{~mm}$ and c) $50 \mathrm{~mm}$.

At the lowest melt-temperature, an increase in the flow rate produces a decrease of the fibre orientation in the flow direction (Figure 8). This is an expected result, since the higher the flow rate the lower the shear stresses experienced by the flow during the filling stage of injection moulding. This is in qualitative agreement with results published by Lian in poly(methyl methacrylate) rectangular plates and by $\mathrm{Bay}^{1,3}$ in $43 \%$ glass fibre reinforced polyamide 6.6. A similar trend may be observed in simulation results, compare the $\mathrm{V}$ shape in Moldflow predictions at the midplane of Figure $8-\mathrm{a}, \mathrm{b}$ and $\mathrm{c}$.

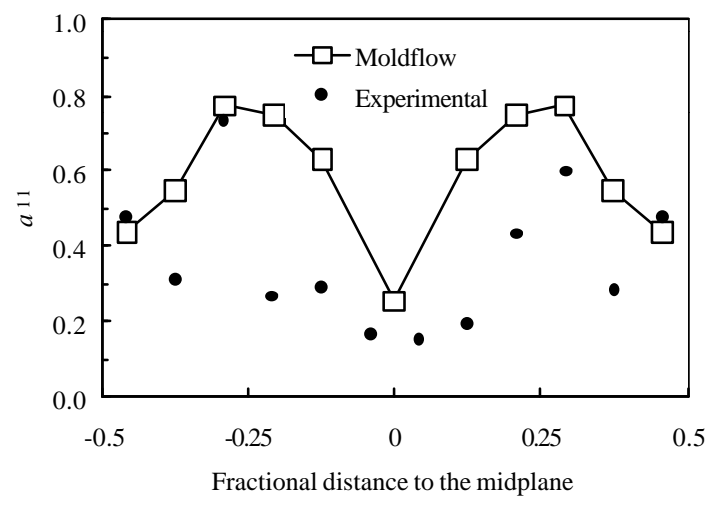

a)

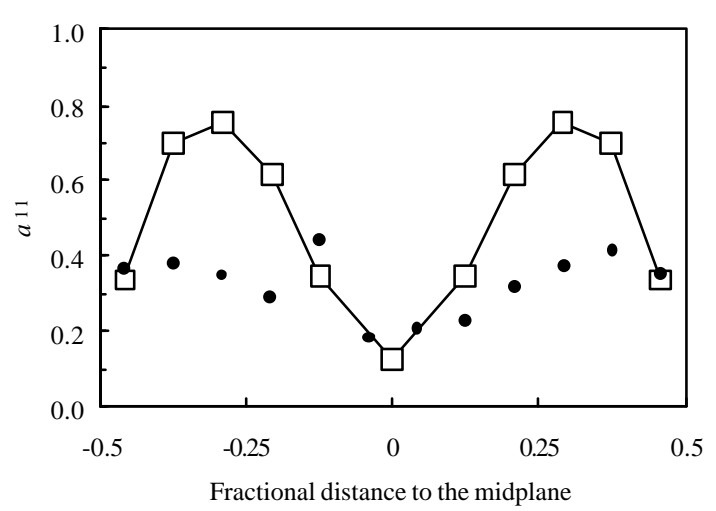

c)

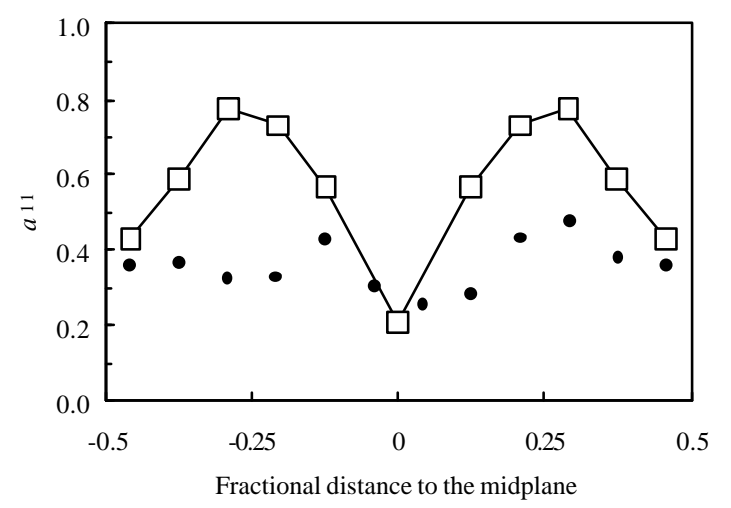

b)

Figure 8 Fibre orientation in the flow direction at $280^{\circ} \mathrm{C}$ melt temperature and at $35 \mathrm{~mm}$ from the gate, at different flow rates a) $10 \mathrm{~cm}^{3} / \mathrm{s}$, b) $14 \mathrm{~cm}^{3} / \mathrm{s}$ and c) $32 \mathrm{~cm}^{3} / \mathrm{s}$. 
Journal Reinforced Plastics and Composites, vol. 20: $n^{\circ} 6$ (2001), p.452-465.

The fibre orientation in flow direction in most of the points is overpredicted by the simulations. The deviations are larger in the intermediate through-thickness region. Close to the wall and at the core of the predicted fibre orientation is in good agreement with experimental results. This is an indication that the shear flow behaviour is less well modelled than the extensional flow behaviour in terms of fibre orientation.

At $320^{\circ} \mathrm{C}$ melt-temperature (Figure 9) it can be observed that the experimental fibre orientation in the flow direction is very small, corresponding to very high tangential fibre orientation. In the simulations, a very different result is obtained, with high fibre orientation in flow direction.

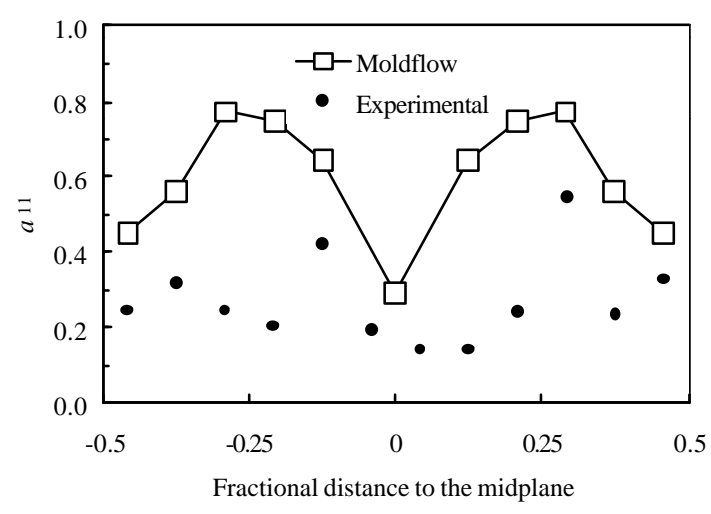

a)

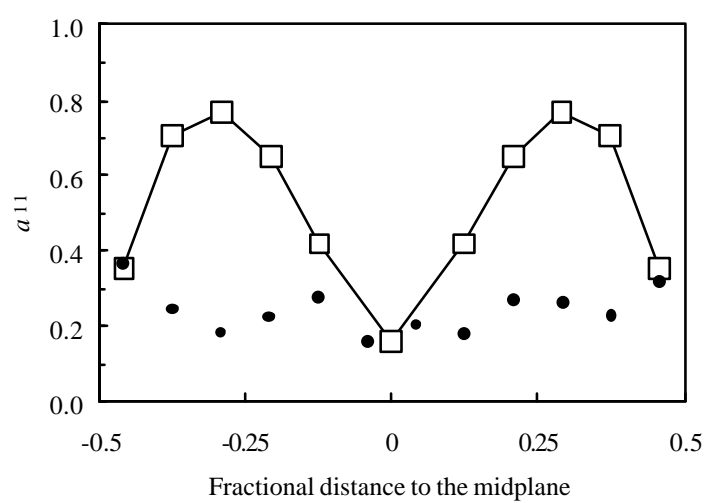

c)

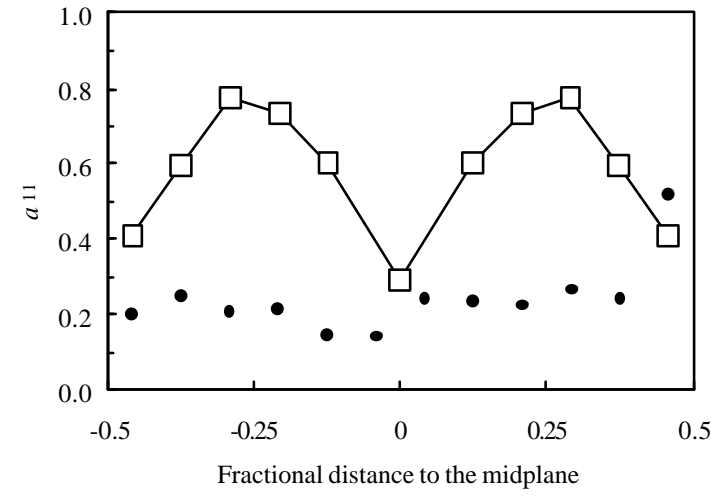

b)

Figure 9 Fibre orientation in flow direction at $320^{\circ} \mathrm{C}$ melt temperature and at $35 \mathrm{~mm}$ from the gate, at different flow rates a) $10 \mathrm{~cm}^{3} / \mathrm{s}$, b) $14 \mathrm{~cm}^{3} / \mathrm{s}$ and c) $32 \mathrm{~cm}^{3} / \mathrm{s}$.

The effect of increasing the flow rate from 10 to $32 \mathrm{~cm}^{3} / \mathrm{s}$ at $320^{\circ} \mathrm{C}$ melt-temperature is very small both in the simulations and in the experimental results.

The effect of increasing the melt temperature at $14 \mathrm{~cm}^{3} / \mathrm{s}$ flow rate is hardly noticeable both in experimental results and in the simulations (Figure 10). This insensitivity to the melt-temperature was already predicted by Bay ${ }^{1}$. 


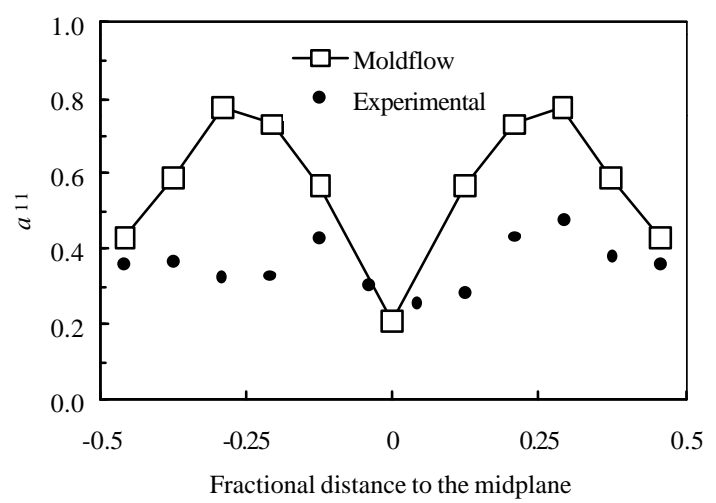

a)

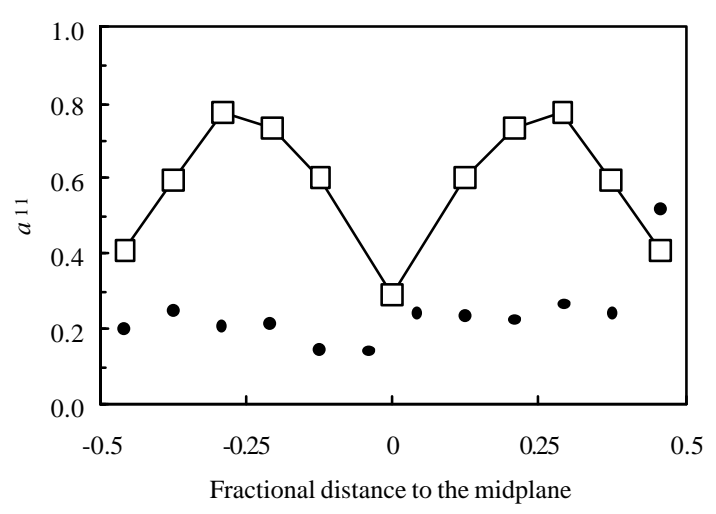

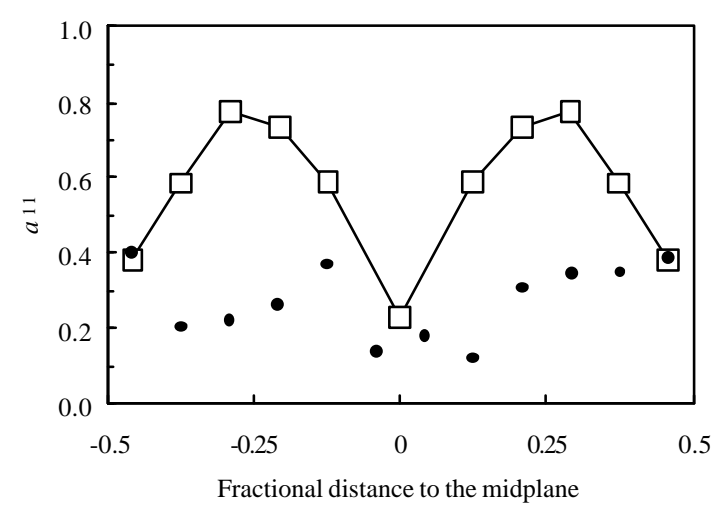

b)

c)

Figure 10 Fibre orientation in flow direction at $35 \mathrm{~mm}$ from the gate with flow rate $14 \mathrm{~cm}^{3} / \mathrm{s}$, and different melt temperature a) $280^{\circ} \mathrm{C}$, b) $300^{\circ} \mathrm{C}$ and c) $320^{\circ} \mathrm{C}$.

The simulation software also has a main parameter to be adjusted in Moldflow modelling is the interaction coefficient. The results indicate that the empirical equation (4), proposed by Tucker, do not give a reliable estimate of the parameter $C_{I}$ for this low volume fibre content material.

It can also be argued that the program overpredicts the shear stresses developing in this geometry for this material. The extensional stresses, which are more effective upon aligning the fibres, are probably responsible for the very low fibre orientation in flow direction.

\section{Conclusions}

The Moldflow predictions of fibre orientation distribution in through-thickness in discs moulded with a $10 \%$ glass fibre reinforced polycarbonate are somehow disappointing.

At the centreline of the disc and close to the wall, the predictions of fibre orientation are in most of the moulding conditions close to the experimental results. At the intermediate points, the fibre orientation in the flow direction is consistently overestimated.

This may be explained by the shear stresses developing in injection moulding being smaller than predicted by the software or, by an overestimation of the interaction between neighbouring fibres.

A qualitative agreement was observed between the predictions and the experimental results in terms of the effect of the moulding conditions over the fibre orientation distribution.

Qualitative agreement was observed in terms of the evolution of fibre orientation along the flow path. Quantitative agreement between predictions and experimental fibre orientation results was observed close to the wall and at the midplane. 
Journal Reinforced Plastics and Composites, vol. 20: $n^{\circ} 6$ (2001), p.452-465.

\section{References}

Advani, S. G., Tucker III, C. L., J. of Rheology, v. 31, n. 8, p. 751-784, 1987.

Akbar, S. and Altan, M. C., Polym. Eng. and Sci., v. 32, n. 12, p. 810-822, 1992.

Batchelor, G. K., J. of Fluid Mech., v. 41, n. 31, p. 545-570, 1970

Batchelor, G. K., J. of Fluid Mech., v. 46, n. 4, p. 813-829, 1971.

Bay $^{1}$, R. S., Tucker III, C. L., Polymer Composites, v. 13, n. 4, p. 332-341, 1992.

Bay $^{2}$, R. S., Tucker III, C. L., Polym. Eng. and Sci., v. 32, n. 4, p. 240-253, 1992.

Bay $^{3}$, R. S., Tucker III, C. L., Davis, R. B., SPE Antec Tech. Papers, p. 539, 1989.

Cox, R. G., J. of Fluid Mech., v. 44, n. 4, p. 791-810, 1970.

Folgar, F. and Tucker III, C. L., J. of Reinf. Plast. and Comp., v. 3, p. 98-119, 1984.

Jeffery, G. B., Proc. Roy. Soc., A102, p. 161-179, 1922.

Lian, B., Ladewig, J., Wille, J. M., McGrath, J. J., SPE Antec Tech. Papers, p. 2316, 1994.

Möginger, B., Eyerer, P., Composites, v. 22, n. 5, p. 394-9, 1991.

Tucker III, C. L., Advani, S. G., Processing of Short-Fiber Systems, in S. G. Advani, ed., Flow and Rheology in Polymer Composites Manufacturing, Comp. Mater. Series, Amsterdam, p. 147-202, 1994. 